TITLE:

\title{
A new index for precise design and advanced operation of mass transfer in slug flow
}

$\operatorname{AUTHOR}(\mathrm{S})$ :

Aoki, Nobuaki; Tanigawa, Shin; Mae, Kazuhiro

\section{CITATION:}

Aoki, Nobuaki ... [et al]. A new index for precise design and advanced operation of mass transfer in slug flow. Chemical Engineering Journal 2011, 167(2-3): 651-656

ISSUE DATE:

2011-03

URL:

http://hdl.handle.net/2433/157341

\section{RIGHT:}

(c) 2010 Elsevier B.V.; この論文は出版社版でありません。引用の際には 出版社版をご確認ご利用ください。; This is not the published version. Please cite only the published version. 
Original Paper

A new index for precise design and advanced operation of mass transfer in slug flow

Nobuaki Aoki, Shin Tanigawa, Kazuhiro Mae

Department of Chemical Engineering, Graduate School of Engineering, Kyoto

University, Kyoto-daigaku Katsura, Nishikyo-ku, Kyoto 615-8510, Japan

*Corresponding author. Tel.: +81 75383 2668; fax: +81 753832658.

E-mail address: kaz@cheme.kyoto-u.ac.jp (K. Mae). 


\section{Abstract}

Slug flow, one of the ordered multi-phase flow patterns in a small channel, has the advantage of the enhancement of mixing in each phase and mass transfer between two phases due to the internal circulation flow. To form stable slug flow, the throughput of order of $\mu \mathrm{L} \cdot \mathrm{min}^{-1}$ has been employed. To use slug flow in industrial scale, however, controlled and high throughput mass transfer and an index for design of a channel with slug flow are required. To address this requirement, we examined the influences of operating conditions such as the channel size and the flow rate on flow patterns and the mass transfer rate. Based on the experimental results, we derived an index to represent the mass transfer rate in slug flow. For further improvement of mass transfer in slug flow, we also verified that the channel including a sudden expansion improves the mass transfer efficiency.

Keywords: Slug flow; Microchannel; Mass transfer; Internal circulation flow; Sudden expansion 


\section{Introduction}

Liquid-liquid two-phase flow has been widely used for industrial processes such as organic synthesis and composition separation owing to the mutually immiscible character of two liquids. Mutually immiscible two liquids flow in a horizontal circular tube in various flow patterns as shown in Fig. 1. Charles et al. [1] drew a diagram where flow patterns in a tube with an inner diameter $26 \mathrm{~mm}$ are plotted against each flow rate of two liquids. Nädler et al. [2] and Arirachakaran et al. [3] made similar diagrams for a tube with an inner diameter $59 \mathrm{~mm}$ and $38.1 \mathrm{~mm}$, respectively. However, fewer studies have examined the effects of operating conditions on flow patterns in a channel with a diameter of order from micrometers to several millimeters.

For extraction using liquid-liquid two-phase flow, parallel flow, emulsion, and slug flow have been mainly adopted. In extraction using parallel flow, though stable operation and phase separation after the contact of two phases can be easily achieved, the mass transfer rate is small due to the small interfacial area per a unit volume. Thus, parallel flow is unsuited for industrial processes with high throughput [4]. Mass transfer is rapid in extraction using emulsification because the interfacial area is extremely large. Phase separation, however, takes long time to coalesce stable emulsion since an 
additional operation such as centrifugation or integrating a device for emulsion coalescence [5] is required. Extraction using emulsion is, hence, suited for the process only requiring rapid extraction.

Extraction using slug flow is based on molecular diffusion, usually slow, the same as in parallel flow. The interfacial area for mass transfer of slug flow is not as large as that of emulsion. Nevertheless, rapid mass transfer between two phases is achieved because it is enhanced by the circulation flow inside a slug generated by the shear between fluid and the channel wall. There have been many reports investigating flow patterns inside a slug in detail experimentally or with simulation [6,7]. Fluid flows forwards at the center of the channel and backwards near the channel wall and circulates inside a slug. The circulation flow enhances mixing inside a slug, making the concentration gradient gradual and also renews fluid (i.e. the solute concentration) close to the interface of two phases, improving the mass transfer rate through the interface.

According to conventional theories, liquid-liquid extraction depends on the mass transfer flux (the product of the mass transfer coefficient and the concentration gradient in simple form), the interfacial area, and residence time. Kashid et al. [8,9] have investigated the mass transfer efficiency in slug flow in terms of the mass transfer coefficient. The interfacial area used for mass transfer depends on both the slug length 
and the thickness of liquid film formed between a slug and the channel wall. Trachsel et al. [10] have reported the narrow residence time distribution of fluid within each slug due to restricted mixing along the channel axis and the possibility of controlling residence time. In addition, phase separation after extraction is achieved easily because segments of fluid in slug flow are large compared to emulsion [4]. Owing to the advantages mentioned above, slug flow is expected to be useful way for controlling mass transfer and slug flow has been applied for reactions including mass transfer $[11,12]$.

To form stable slug flow, however, the throughput of order of $\mu \mathrm{L} \cdot \mathrm{min}^{-1}$ and channels with diameters of micrometers or millimeters have mainly been employed while much higher order of flow rate is required to use slug flow in industrial scale. We also have to determine operating parameters such as the channel size, the flow rate of each phase, and the flow rate ratio of two phases for obtaining a slug flow operation giving a desired mass transfer rate. However, few studies correlate these variables with the extraction efficiency or the mass transfer coefficient to establish a design guideline of slug flow operation. To satisfy these requirements, this research addresses the effects of operating conditions on flow patterns and the mass transfer efficiency and then establishes an index for choosing operating conditions with a desired mass transfer rate 
in slug flow. For further improvement of mass transfer in slug flow, we also examine the effects of channel shape of sudden expansion and contraction.

\section{Experimental}

\subsection{Flow patterns of two-phase flow}

We visualized two-phase flow of water and dodecane to identify the range of experimental conditions under which slug flow is stably formed. The schematic of experimental set-up is shown in Fig. 2. PTFE tubes with an inner diameter $d$ and a length $L$ were connected to the entrances and the exit of a union tee (Swagelok) made of SUS with an inner diameter $d_{\text {unionT. }}$ Distilled water colored by red ink and dodecane were supplied to a union tee by syringe pumps (IC3210, Kd Scientific and PHD4400Hpsi, Harvard Apparatus) through PTFE tubes. Table 1 shows $d$ and $d_{\text {unionT }}$ of $1 / 16 ", 1 / 8$ ", and $1 / 4$ " union tees. Flow pattern in a PTFE tube attached to the exit of a union tee was observed with varying total flow rates of the two phases, $V_{\mathrm{t}}$, and ratio of the water phase flow rate to the total flow rate, $w f$. 


\subsection{Extraction}

We performed extraction of phenol in dodecane to water phase as a model system to evaluate the mass transfer efficiency in slug flow. The experimental set-up is the same as Fig. 2. Distilled water was fed into an entrance of a union tee, and dodecane dissolving phenol (1000 ppm) into the other. The flow rates of the two fluids were equal, that is, $w f=0.5$. Phenol is extracted from dodecane phase to water phase in slug flow. Fluid from the exit was sampled in a test tube for $20 \mathrm{~s}$. The two phases immediately separate in a test tube. The concentration of extracted phenol in water phase, $C$, was measured from the UV absorbance at $270 \mathrm{~nm}$ using an ultraviolet and visible spectrometer (Shimadzu, Multispec-1500). In this experiment, $d, L, d_{\mathrm{unionT}}$, and $V_{\mathrm{t}}$ were varied. The total flow rate, $V_{\mathrm{t}}$ was 10 and $20 \mathrm{~mL} \cdot \mathrm{min}^{-1}$ for all channel sizes, and 30 $\mathrm{mL} \cdot \mathrm{min}^{-1}$ for $1 / 8$ " union tee. In this range of $V_{\mathrm{t}}$, we confirmed that stable slug flow was formed in a tube of each size. Sampling and measuring the concentration of phenol were repeated three or four times for each experimental condition, and the average values of $C$ were used to calculate the mass transfer rate in section 3.2. Lengths of water phase slugs in the exit channel, $l$, were also measured with calipers and the average values 
were used.

\subsection{Channel expansion and contraction}

We performed extraction using a channel with a channel expansion or contraction in the course of the exit channel to investigate the effect of these channel shapes on the mass transfer efficiency. For a channel with expansion, a PTFE tube with an inner diameter $1.00 \mathrm{~mm}$ and a length $L_{1}$ was attached to the exit of a $1 / 16$ " union tee, and to the end of the tube another PTFE tube with an inner diameter $1.59 \mathrm{~mm}$ and a length $L_{2}$ was connected as shown in Fig. 3(a). For a channel with a channel contraction, a PTFE tube with an inner diameter $1.59 \mathrm{~mm}$ and a length $10 \mathrm{~cm}$ was attached to the exit of a $1 / 8$ " union tee, and to the end of the tube another PTFE tube with an inner diameter $1.00 \mathrm{~mm}$ and a length $10 \mathrm{~cm}$ was connected, as shown in Fig. 3(b). At first, the total tube length was fixed at $20 \mathrm{~cm}$ and $L_{1}=L_{2}=10 \mathrm{~cm}$. The flow rates of the two phases were equal and $V_{\mathrm{t}}=10$ and $20 \mathrm{~mL} \cdot \mathrm{min}^{-1}$, corresponding to the total residence time, $\tau=1.7 \mathrm{~s}$ and $0.83 \mathrm{~s}$, respectively. The extraction efficiency in two channels were evaluated and compared using the extraction ratio, $\xi$, expressed as $\xi=$ Phenol transferred to water phase / Phenol initially fed into dodecane phase 
Then the effect of the location of the channel expansion on the mass transfer efficiency was examined. The location of the channel expansion was varied by changing $L_{1}$ and $L_{2}$ so that the total residence time was fixed at $0.83 \mathrm{~s}$. The extraction ratio, $\xi$, was employed to evaluate the results.

\section{Results and discussion}

\subsection{Stability of slug flow}

Distributions of flow patterns against the total flow rate and the flow rate ratio of water phase for 1/16" and 1/8" union tees are shown in Fig. 4 (see also Fig. 1 for flow patterns). For a $1 / 16$ " union tee, stable slug flow was observed in the range of $V_{\mathrm{t}}$ under $10 \mathrm{~mL} \cdot \mathrm{min}^{-1}$ for all the $w f$, and $V_{\mathrm{t}}$ under $20 \mathrm{~mL} \cdot \mathrm{min}^{-1}$ for $w f=0.5$, excepting when $V_{\mathrm{t}}$ was extremely small. Stable slug flow was formed in the range of $V_{\mathrm{t}}$ up to 20 $\mathrm{mL} \cdot \mathrm{min}^{-1}$ for all the $w f$ for a $1 / 8$ " union tee. For $1 / 4$ " union tees $(d=3.17 \mathrm{~mm}$ and 4.35 $\mathrm{mm}$ ), stability of slug flow was also confirmed in the range of $V_{\mathrm{t}}$ less than or equal to 30 $\mathrm{mL} \cdot \mathrm{min}^{-1}$. To sum up, the maximum flow rate forming stable slug flow increases with a rise in channel diameter. Moreover, slug flow can be formed under flow rates in the 
range of several tens of $\mathrm{mL} \cdot \mathrm{min}^{-1}$, which is much higher than those mainly addressed in previous studies.

\subsection{Mass transfer efficiency}

\subsubsection{Calculation of the mass transfer rate}

The mass transfer rate was calculated to assess the mass transfer efficiency of phenol from dodecane phase to water phase. The temporal change of the concentration of extracted phenol in water phase is expressed as

$$
\mathrm{d} C / \mathrm{d} t=k a\left(C^{*}-C\right)
$$

or in integrated form,

$$
\ln \left\{C^{*} /\left(C^{*}-C\right)\right\}=k a \tau
$$

where $C^{*}$ is the equilibrium extract concentration of phenol in water phase, $k$ is the overall mass transfer coefficient in water phase, $a$ is the specific surface area of a slug of water phase, $t$ is the time, and $\tau$ is the residence time at the exit channel. The values of $C^{*}$ for $w f=0.3,0.5$, and 0.7 are shown in Table 2. In Eq. (3), $\ln \left\{C^{*} /\left(C^{*}-C\right)\right\}$ is proportional to the residence time, $\tau$ with the mass transfer rate, $k a$, as a proportional constant. The residence time, $\tau$ is defined by $V_{\mathrm{t}}, d$, and $L$. 


$$
\tau=4 L / \pi d^{2} V_{\mathrm{t}}
$$

The mass transfer efficiency depends on $V_{\mathrm{t}}$ and $d$, but not on $L$. Therefore, for obtaining the value of $k a, \ln \left\{C^{*} /\left(C^{*}-C\right)\right\}$ was plotted against $\tau$. The value of $k a$ is obtained from the slope of the fitted line in the range of small $\tau$.

\subsubsection{Effect of the slug velocity}

The effect of the slug velocity on the mass transfer efficiency was investigated with a fixed channel size $(1 / 8$ " union tee) and ratio of water phase $(w f=0.5)$. The temporal change of the function of the extract concentration, $\ln \left\{C^{*} /\left(C^{*}-C\right)\right\}$, for each $V_{\mathrm{t}}$ is shown in Fig. 5. The value of $\ln \left\{C^{*} /\left(C^{*}-C\right)\right\}$ increases with residence time, $\tau$, when $\tau$ is relatively small. However, the increase in $\ln \left\{C^{*} /\left(C^{*}-C\right)\right\}$ is small at higher values of $\tau$. This tendency indicates that the mass transfer proceeds intensively just after the contact of the two phases; then the mass transfer enhanced by the internal circulation flow falls off; and finally molecular diffusion dominates the mass transfer. Fig. 6 shows the best fit lines for the data in small $\tau$ range and calculated $k a$ values. In the following section, the same method is used to obtain $k a$. To make the influence of the slug velocity clear, $k a$ is plotted against the slug velocity, $U$, calculated from $V_{\mathrm{t}}$ and $d$, in Fig. 7. The slug lengths of water phase, $l$, measured for each slug velocity are also given in the 
diagram. The mass transfer rate, $k a$, increases with a rise in slug velocity. The increase in slug velocity enhances the internal circulation flow rate in a slug and also shortens the slug length. These effects raise the frequency of the internal circulation.

\subsubsection{Effect of the channel size}

The effect of the channel size was investigated with a fixed total flow rate $\left(V_{\mathrm{t}}=\right.$ $\left.20 \mathrm{~mL} \cdot \mathrm{min}^{-1}\right)$ and ratio of water phase $(w f=0.5)$. To specify the effect of the channel size, $k a$ is plotted against the channel diameter in Fig. 8. The water slug length measured for each channel size is also given in the diagram. The slug length goes up with increasing $d$, excepting the point for $d=4.35 \mathrm{~mm}$. It was confirmed that $\mathrm{ka}$ increases as the channel size decreases. The reasons for this tendency are explained as follows. First, the decrease in channel diameter increases the velocity that slugs move at a fixed total flow rate. As a result, the velocity of the internal circulation flow increases. In addition, the slug diameter and length decrease, resulting in a reduced length of circulation path inside slugs. These effects lead to the enhancement of the internal circulation frequency.

\subsubsection{Derivation of the turnover index of the mass transfer efficiency}


The effects of the slug velocity and the channel size on the mass transfer rate have been described in sections 3.2.2 and 3.2.3. The results in these sections reveal that the slug length varies with the change of the slug velocity or channel size and that the slug size (diameter and length) also influences the mass transfer rate. Thus, to arrange the effects of these manipulated variables on the mass transfer rate, we derived an index consisting of these variables, denoted by $U /(d+2 l)$. The length denoted by $d+2 l$ represents the length of circulation path for the internal circulation flow inside a slug as shown in Fig. 9. The value of $U /(d+2 l)$ represents the turnover frequency of fluid in a slug. Fig. 10 shows the relationship between the turnover index, $U /(d+2 l)$, and the mass transfer rate, $k a$. The value of $k a$ improves with increasing $U /(d+2 l)$. The increase in the slug velocity $(U)$ and decrease in the slug size ( $d$ and $l$ ) leads to a higher value of the index, so this tendency is consistent with the results in the previous sections. The circulation flow inside a slug renews the concentration at the phase interface more efficiently with a higher $U /(d+2 l)$. In addition, the plots of $k a$ are on the same line regardless of the channel size. Therefore, we can determine the channel size and the flow rate to obtain a desired mass transfer rate by choosing an appropriate value of the turnover index, $U /(d+2 l)$. The effect of physical property of fluids such as viscosity is included in the slug length. Therefore, this index is expected to be effective in different 
kinds of fluids.

\subsubsection{Effect of the additional channel expansion and contraction}

Fig. 11 shows the extraction ratio in the channels with an additional expansion and contraction for the total flow rates, $V_{\mathrm{t}}=10$ and $20 \mathrm{~mL} \cdot \mathrm{min}^{-1}$. The extraction ratio in the channel with the expansion is higher than that with contraction for the both total flow rates. This indicates that the channel with the expansion shows better mass transfer efficiency.

Fig. 12 shows the relationship between the location of the channel expansion and the extraction ratio. At the right end, $L_{2}=0 \mathrm{~cm}$, the channel does not include a channel expansion, and the channel diameter is fixed at $1.00 \mathrm{~mm}$. At the left end, $L_{1}=0$ $\mathrm{cm}$, the channel has no expansion and consists of a $1 / 8$ " union tee, and a channel with a diameter $1.59 \mathrm{~mm}$. The extraction ratio maximizes at $L_{1}=20 \mathrm{~cm}$ and $L_{2}=6 \mathrm{~cm}$. This indicates that the mass transfer efficiency can be improved by adding a channel expansion. The reason for this tendency is inferred as follows: since the liquid film is thin, almost no oil phase fluid exists in the sides of water phase slugs. At the channel expansion, the sides as well as the front and back of water phase slugs are surrounded by oil phase, resulting in an increased contact area of the two phases. However, after 
this point, the surface area to volume ratio decreases because of the expanded channel size. Thus, an additional reason is needed to justify this tendency. The change in slug length is also an important factor in the interpretation. When the channel is expanded, the slug length is shortened with a fixed slug volume. This leads to a short length of circulation path in the axial direction inside slug. For example, when the slug diameter $d$ is changed to $2 d$, the slug length $l$ is reduced to $0.25 l$. The length of circulation path is changed from $d+2 l$ into $2 d+0.25 l$. Usually, $l$ is larger than $d$. Therefore, the length of circulation path is reduced with the expansion. This is also the reason why the expansion gives the better mass transfer rate than the contraction.

The advantage of the internal circulation flow over mass transfer efficiency seems avail in an early stage after the contact of the two phases as described in sections 3.2.2 and 3.2.3. This means that mass transfer in the channel near the exit is slow in slug flow. Therefore, setting an additional channel expansion is an effective method to reactive mass transfer in the channel near the exit.

\section{Conclusions}

We have investigated a methodology to design a channel and to determine 
operating conditions for extraction using slug flow with the throughput of several tens of $\mathrm{mL} \cdot \mathrm{min}^{-1}$ order. Based on the experimental results, we derived the turnover index including the slug velocity and size that means the turnover frequency of the internal circulation flow. The relationship between the mass transfer rate and the turnover index implies that the mass transfer rate can be represented by the index. Therefore, the turnover index enables us to determine arbitrarily a set of operating conditions to achieve the desired mass transfer rate.

For further enhancement of mass transfer in slug flow, the effects of an additional channel expansion or contraction has also been examined. A channel with a channel expansion shows better extraction rate than that with a contraction. In addition, the extraction efficiency maximizes at the specific location of the channel expansion in the exit channel. This indicates that the mass transfer efficiency can be improved by utilizing a sudden change of the channel diameter, especially in the channel near the exit, where the advantage of the internal circulation flow is reduced.

\section{Notation}

$a$

$C$
Specific surface area of a water slug $\left(\mathrm{m}^{-1}\right)$

Concentration of extracted phenol in water phase $\left(\mathrm{mol} \cdot \mathrm{m}^{-3}\right)$ 


$\begin{array}{ll}C^{*} & \text { Equilibrium extract concentration of phenol in water phase }\left(\mathrm{mol} \cdot \mathrm{m}^{-3}\right) \\ d & \text { Inner diameter of a channel of a PTFE tube }(\mathrm{m}) \\ d_{\text {unionT. }} & \text { Inner diameter of a union tee }(\mathrm{m}) \\ l & \text { Slug length of water phase }(\mathrm{m}) \\ L, L_{1}, L_{2} & \text { Length of a channel of a PTFE tube }(\mathrm{m}) \\ k & \text { Overall mass transfer coefficient in water phase }\left(\mathrm{m} \cdot \mathrm{s}^{-1}\right) \\ t & \text { Time (s) } \\ & \text { Slug velocity }\left(\mathrm{m} \cdot \mathrm{s}^{-1}\right) \\ U & \text { Total flow rate }\left(\mathrm{mL} \cdot \mathrm{min}^{-1}\right) \\ V_{\mathrm{t}} & \end{array}$

Greek letters

$\xi \quad$ Extraction ratio (-)

$\tau \quad$ Residence time (s)

\section{Acknowledgement}

This research was supported by the New Energy and Industrial Technology 
Development Organization (NEDO), Project of Development of Microspace and Nanospace Reaction Environment Technology for Functional Materials.

\section{References}

[1] M.E. Charles, G.W. Govier, G.W. Hodgson, The horizontal pipeline flow of equal density oil-water mixtures, Can. J. Chem. Eng. 39 (1961) 27-36.

[2] M. Nädler, D. Mewes, The effect of gas injection on the flow of immiscible liquids in horizontal pipes, Chem. Eng. Thechnol. 18 (1995) 156-165.

[3] S. Arirachakaran, K.D. Oglesby, M.S. Malinowsky, O. Shoham, J.P. Brill, An Analysis of Oil/Water Flow Phenomena in Horizontal Pipes, Paper SPE 18836, Society of Petroleum Engineers, Oklahoma, 1989, pp. 155-167.

[4] Y. Okubo, T. Maki, N. Aoki, T.H. Khoo, Y. Ohmukai, K. Mae, Liquid-liquid extraction for efficient synthesis and separation by utilizing micro spaces, Chem. Eng. Sci. 63 (2008) 4070-4077.

[5] Y. Okubo, M. Toma, H. Ueda, T. Maki, K. Mae, Microchannel devices for the coalescence of dispersed droplets produced for use in rapid extraction process, Chem. Eng. J. 101 (2004) 39-48. 
[6] M.N. Kashid, I. Gerlach, S. Goetz, J. Franzke, J.F. Acker, F. Platte, D.W. Agar, S. Turek, Internal circulation within the liquid slugs of a liquid-liquid slug-flow capillary microreactor, Ind. Eng. Chem. Res. 44 (2005) 5003-5010.

[7] W. Tanthapanichakoon, N. Aoki, K. Matsuyama, K. Mae, Design of mixing in microfluidic liquid slugs based on a new dimensionless number for precise reaction and mixing operations, Chem. Eng. Sci. 61 (2006) 4220-4232.

[8] M.N. Kashid, Y.M. Harshe, D.W. Agar, Liquid-liquid slug flow in a capillary: an alternative to suspended drop or film contactors, Ind. Eng. Chem. Res. 46 (2007) $8420-8430$.

[9] M.N. Kashid, D.W. Agar, S. Turek, CFD modelling of mass transfer with and without chemical reaction in the liquid-liquid slug flow microreactor, Chem. Eng. Sci. 62 (2007) 5102-5109.

[10] F. Trachsel, A. Günther, S. Khan, K.F. Jensen, Measurement of residence time distribution in microfluidic systems, Chem. Eng. Sci. 60 (2005) 5729-5737.

[11] J.R. Burns, C. Ramshaw, The intensification of rapid reactions in multiphase systems using slug flow in capillaries, Lab. Chip, 1 (2001) 10-15.

[12] H. Okamoto, Effect of alternating pumping of two reactants into a microchannel on a phase transfer reaction, Chem. Eng. Technol., 29 (2006) 504-506. 


\section{Table and Figure Captions}

Table 1 Channel size

Table 2 Equilibrium concentration of phenol extracted from dodecane phase to water phase

Fig. 1. Examples of flow patterns of liquid-liquid two-phase flow in a horizontal circular tube. (a) Annular flow, (b) Slug flow, (c) Dispersed flow.

Fig. 2. Schematic of the experimental set-up.

Fig. 3. Schematic of a device with a sudden change of the channel diameter. (a) Channel expansion, (b) Channel contraction.

Fig. 4. Flow pattern distributions ( $\circ$ Slug flow uniform in length, $\triangle$ Slug flow not uniform in length, $\times$ Transition range, $\square$ Annular flow). (a) 1/16" union tee, (b) $1 / 8$ " union tee.

Fig. 5. Temporal change of $\ln \left\{C^{*} /\left(C^{*}-C\right)\right\}$ for different slug velocities $\left(1 / 8^{\prime \prime}\right.$ union tee, $\left.w f=0.5, \bullet 5 \mathrm{~mL} \cdot \mathrm{min}^{-1}, \square 10 \mathrm{~mL} \cdot \mathrm{min}^{-1}, \mathbf{\Delta} 20 \mathrm{~mL} \cdot \mathrm{min}^{-1}, \nabla 30 \mathrm{~mL} \cdot \mathrm{min}^{-1}\right)$.

Fig. 6. Best fit lines and $k a$ values for different slug velocities $(1 / 8$ " union tee, $w f=0.5$,

- $\left.5 \mathrm{~mL} \cdot \mathrm{min}^{-1}, \square 10 \mathrm{~mL} \cdot \mathrm{min}^{-1}, \boldsymbol{\Delta} 20 \mathrm{~mL} \cdot \mathrm{min}^{-1}, \nabla 30 \mathrm{~mL} \cdot \mathrm{min}^{-1}\right)$. 
Fig. 7. Effect of the slug velocity on $k a$ and the slug length $(1 / 8$ " union tee, $w f=0.5)$.

Fig. 8. Effect of the channel size on $k a$ and the slug length $\left(V_{\mathrm{t}}=20 \mathrm{~mL} \cdot \mathrm{min}^{-1}, w f=0.5\right)$.

Fig. 9. Characteristic distance for the internal circulation flow to circulate inside a slug.

Fig. 10. Mass transfer rate as a function of the turnover index $(w f=0.5, \bullet 1 / 16$ " union

tee, $\square 1 / 8$ " union tee, $\boldsymbol{\Delta} 1 / 4$ " union tee $(d=3.17 \mathrm{~mm}), \nabla 1 / 4$ " union tee $(d=4.35$

$\mathrm{mm})$ ).

Fig. 11. Comparison of the extraction ratio between the channels with a channel expansion and contraction.

Fig. 12. Relationship between the location of the channel expansion and the extraction ratio. 
Table 1 Channel size

\begin{tabular}{ccc}
\hline Union tee & $d_{\text {unionT }}[\mathrm{mm}]$ & $d[\mathrm{~mm}]$ \\
\hline $1 / 16 "$ & 1.3 & 1.00 \\
$1 / 8 ”$ & 2.3 & 1.59 \\
$1 / 4 "$ & 4.8 & 3.17 \\
$1 / 4 "$ & 4.8 & 4.35 \\
\hline
\end{tabular}


Table 2 Equilibrium concentration of phenol extracted from dodecane phase to water phase

\begin{tabular}{cc}
\hline$w f[-]$ & $C^{*}[\mathrm{ppm}]$ \\
\hline 0.3 & 1879.7 \\
0.5 & 981.0 \\
0.7 & 469.0 \\
\hline
\end{tabular}




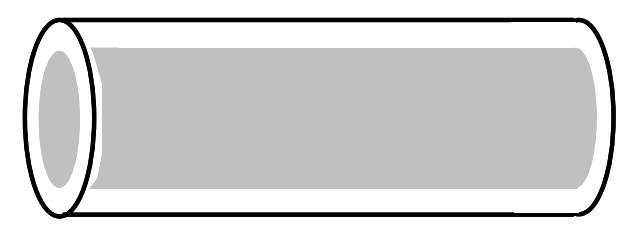

(a) Annular flow

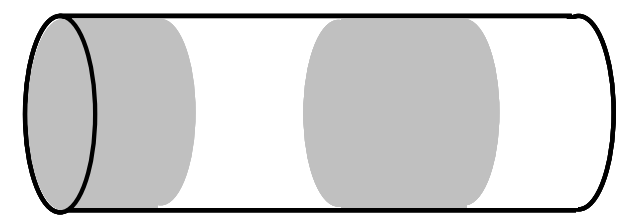

(b) Slug flow

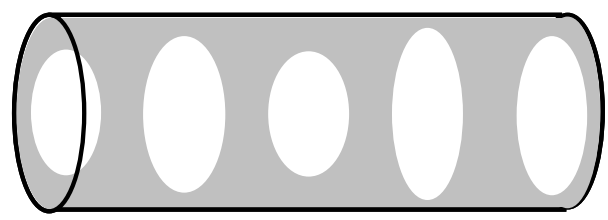

(c) Dispersed flow

Fig. 1. Examples of flow patterns of liquid-liquid two-phase flow in a horizontal circular tube. (a) Annular flow, (b) Slug flow, (c) Dispersed flow. 


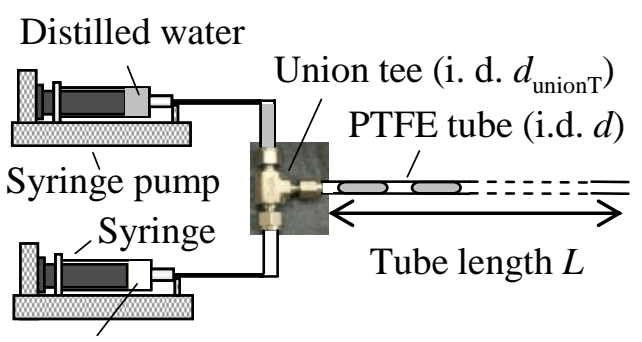

Dodecane

Fig. 2. Schematic of the experimental set-up. 


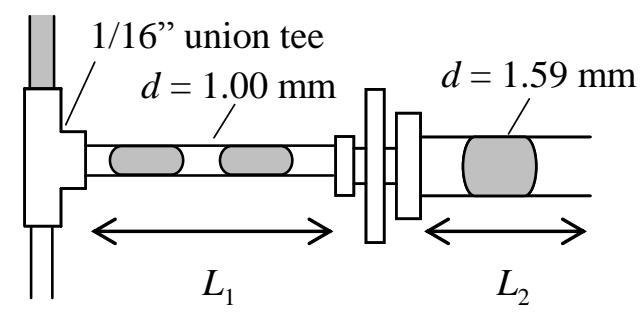

(a) Channel expansion

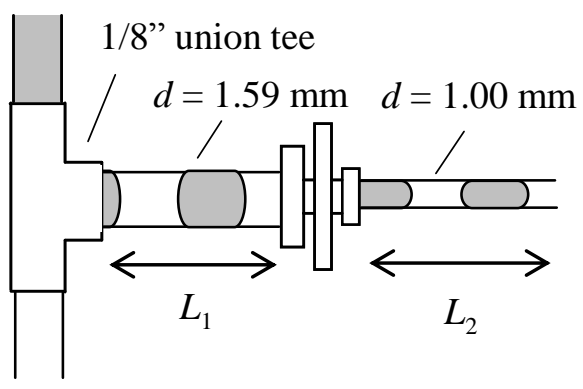

(b) Channel contraction

Fig. 3. Schematic of a device with a sudden change of the channel diameter. (a) Channel expansion, (b) Channel contraction. 


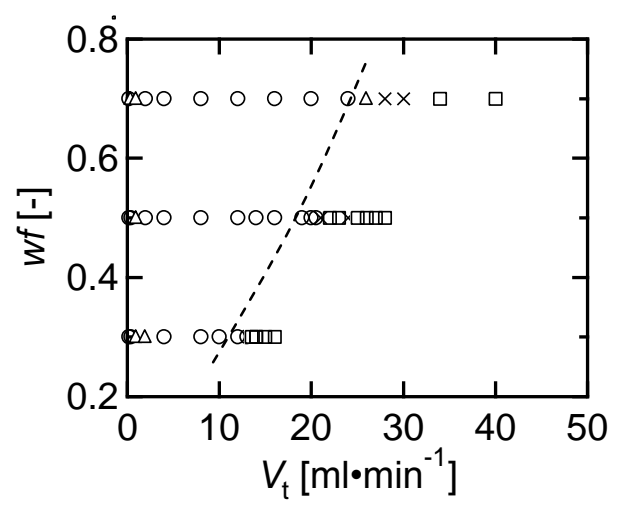

(a) $1 / 16$ " union tee

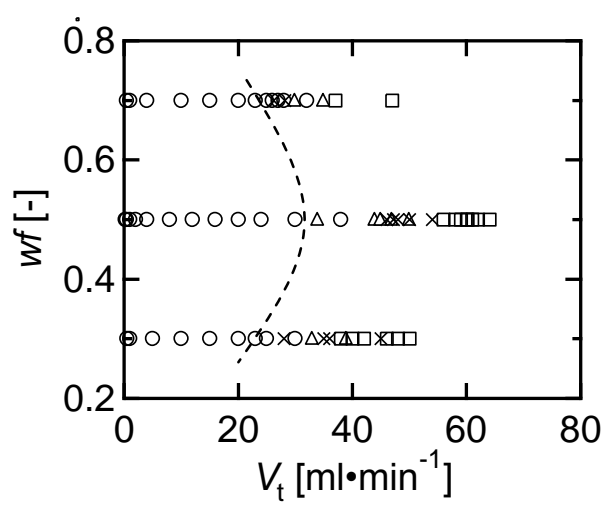

(b) $1 / 8$ " union tee

Fig. 4. Flow pattern distributions ( $\circ$ Slug flow uniform in length, $\triangle$ Slug flow not uniform in length, $\times$ Transition range, $\square$ Annular flow). (a) 1/16" union tee, (b) $1 / 8$ " union tee. 


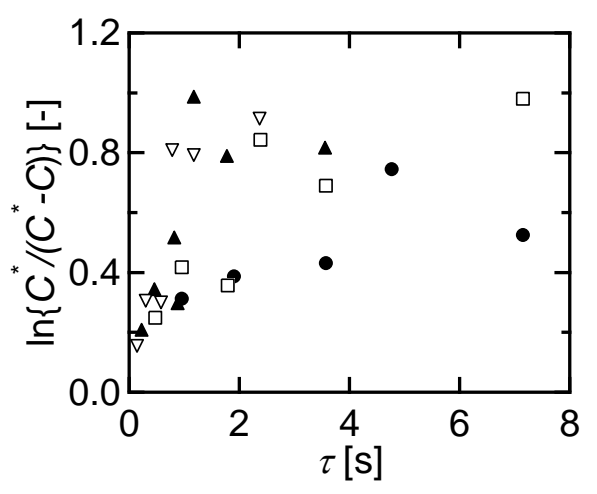

Fig. 5. Temporal change of $\ln \left\{C^{*} /\left(C^{*}-C\right)\right\}$ for different slug velocities $\left(1 / 8^{\prime \prime}\right.$ union tee, $\left.w f=0.5, \bullet 5 \mathrm{~mL} \cdot \mathrm{min}^{-1}, \square 10 \mathrm{~mL} \cdot \mathrm{min}^{-1}, \boldsymbol{\Delta} 20 \mathrm{~mL} \cdot \mathrm{min}^{-1}, \nabla 30 \mathrm{~mL} \cdot \mathrm{min}^{-1}\right)$. 


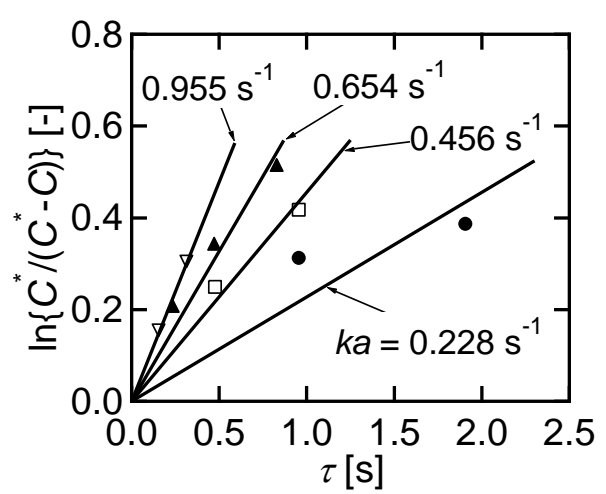

Fig. 6. Best fit lines and $k a$ values for different slug velocities (1/8" union tee, $w f=0.5$,

- $\left.5 \mathrm{~mL} \cdot \mathrm{min}^{-1}, \square 10 \mathrm{~mL} \cdot \mathrm{min}^{-1}, \boldsymbol{\Delta} 20 \mathrm{~mL} \cdot \mathrm{min}^{-1}, \nabla 30 \mathrm{~mL} \cdot \mathrm{min}^{-1}\right)$. 


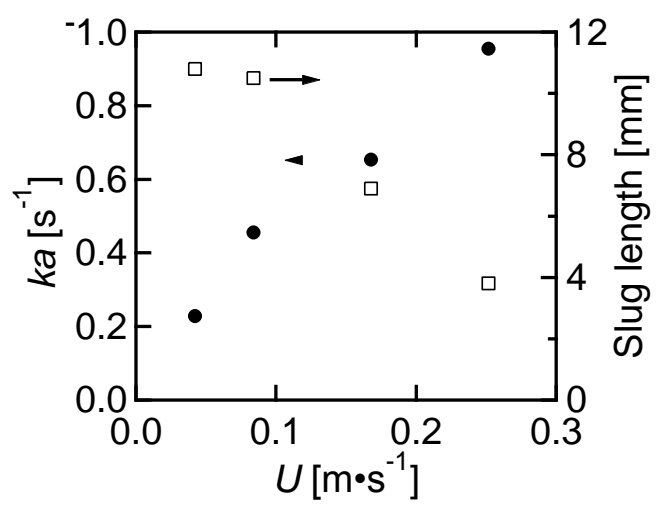

Fig. 7. Effect of the slug velocity on $k a$ and the slug length $(1 / 8$ " union tee, $w f=0.5)$. 


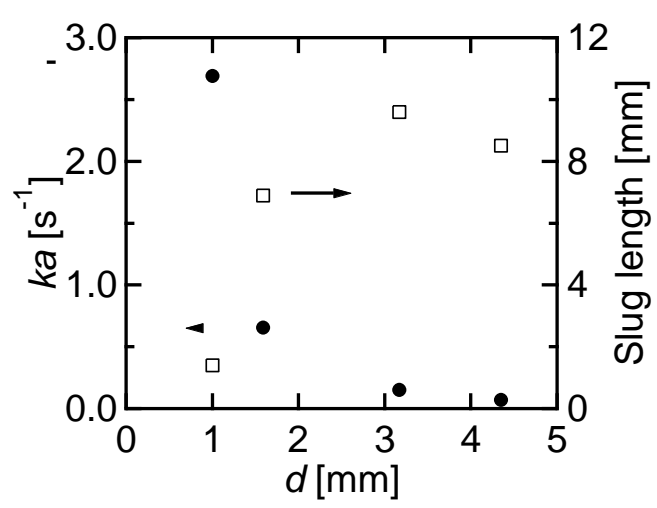

Fig. 8. Effect of the channel size on $k a$ and the slug length $\left(V_{\mathrm{t}}=20 \mathrm{~mL} \cdot \mathrm{min}^{-1}, w f=0.5\right)$. 


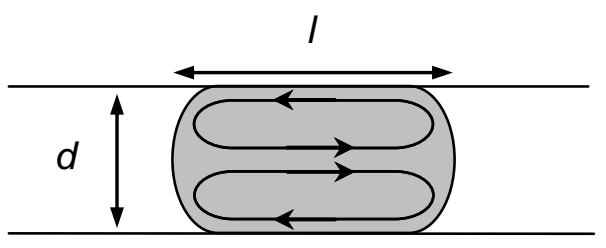

Fig. 9. Characteristic distance for the internal circulation flow to circulate inside a slug. 


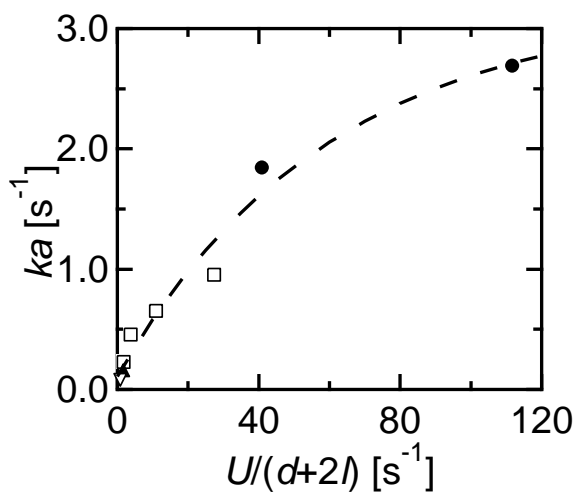

Fig. 10. Mass transfer rate as a function of the turnover index $(w f=0.5, \bullet 1 / 16$ " union tee, $\square 1 / 8$ " union tee, $\boldsymbol{\Delta} 1 / 4$ " union tee $(d=3.17 \mathrm{~mm}), \nabla 1 / 4$ " union tee $(d=4.35$ $\mathrm{mm})$ ). 


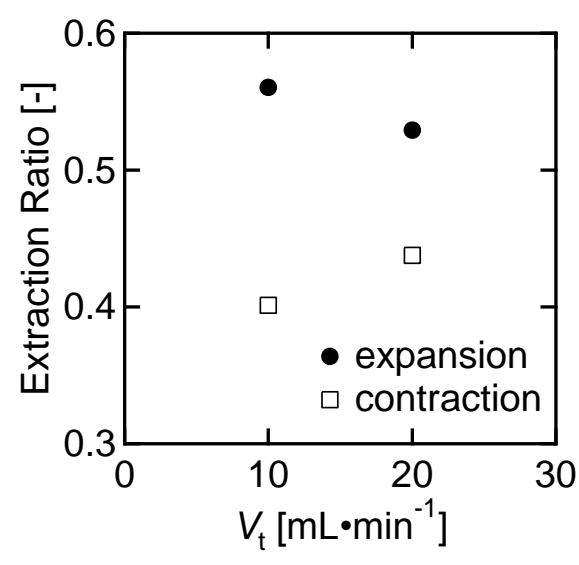

Fig. 11. Comparison of the extraction ratio between the channels with a channel expansion and contraction. 


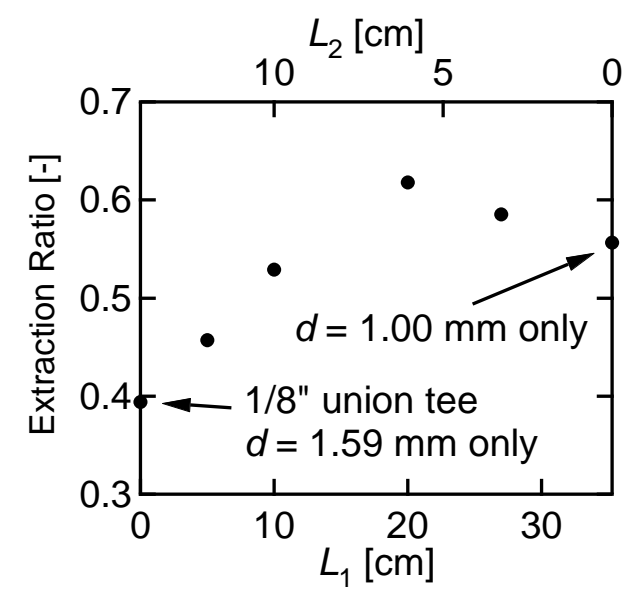

Fig. 12. Relationship between the location of the channel expansion and the extraction ratio. 